\title{
Kanegelo ya Nimirode: Re lebelela diphihlelelo tša gagwe ka lefsa
}

\author{
Author: \\ Elelwani B. Farisani ${ }^{1}$ \\ Affiliation: \\ ${ }^{1}$ Department Old Testament \\ and Ancient Near Eastern \\ Studies, University of South \\ Africa, South Africa \\ Correspondence to: \\ Elelwani Farisani \\ Email: \\ fariseb@unisa.ac.za \\ Postal address: \\ PO Box 392, UNISA 0003, \\ South Africa \\ Dates: \\ Received: 15 Nov. 2010 \\ Accepted: 23 Aug. 2011 \\ Published: 15 Nov. 2011 \\ How to cite this article: \\ Farisani, E.B., 2011, \\ 'Kanegelo ya Nimirode: Re \\ lebelela diphihlelelo tša \\ gagwe ka lefsa', Verbum et \\ Ecclesia 32(1), Art. \#479, \\ 9 pages. http://dx.doi. \\ org/10.4102/ve.v32v1.479 \\ Note: \\ Karolo ya taodišwana ye e \\ phatlaladitšwe la mathomo \\ ka go Farisani, E. 2008. \\ Nimrod: Reading the Bible \\ with South African Eyes, in \\ Pinn A B and Callahan, A \\ D 2008. African American \\ Religious Life and the Story \\ of Nimrod. New York: \\ Palgrave Macmillan, 97-109. \\ E dirišitšwe mo ka tumelelo \\ ya Palgrave Macmillan. \\ Taodišwana ye e tšwela pele \\ ka go tsentšha tshedimošo \\ ye mpsha ye e sego gona mo \\ phatlalatšong ya Palgrave \\ Macmillan.
}

(C) 2011. The Authors. Licensee: AOSIS OpenJournals. This work is licensed under the Creative Commons Attribution License.

\section{The Nimrod story: Reconsidering his achievements}

The Ham/Nimrod story has been used to oppress Black people in South Africa and the USA. This paper looks afresh at Nimrod and attempts to restore his dignity and that of black people. This will be done in five stages below. Firstly, we briefly highlight the identity of Noah's children in order to place into context Nimrod, who is the focus of this study. Secondly, we examine the negative portrayal of Nimrod by certain scholars. Thirdly, we discuss the use of Ham/Nimrod's story in the oppression or enslavement of black people. Furthermore, we will critique such a use of the story to oppress black people. Fourthly we discuss Nimrod's achievements. The focus here will be on his three major achievements as a great ruler, mighty hunter and builder. The aim of this section is to counter the negative interpretation that Nimrod has previously received. In addition, we will offer a positive interpretation of the curse on Ham/Cush/Nimrod. Fifth and, finally, we will spell out the significance of the Nimrod story for those who have been oppressed and enslaved on the basis of this text. The focus here will be on South Africa.

\section{Matseno}

Kanegelo ya Hama/Nimirode e šomišitšwe go gatelela Bathobaso ba Afrika-Borwa le ba Amerika. Taodišwana ye e lekola Nimirode ka lefsa le go tšea magato a go bušetša seriti sa gagwe le sa Bathobaso sekeng.

Seo se tlo dirwa ka dikgato tše tlhano ka mo tlase. Sa mathomo, re tlo lekola dimelo tša bana ba Noage, go kwešiša Nimirode, yo e lego yena šedi ye kgolo ya taodišwana ye. Sa bobedi re tlo lebelela ka fao bangwadi ba bangwe ba tšweletšago seswantšho sa go befa sa Nimirode. Sa boraro, re tlo sekaseka tirišo ya kanegelo ya Hama/Nimirode go gatelela le go dira Bathobaso makgoba. Sa bone, re tlo sekaseka diphihlelelo le dikatlego tša Nimirode. Mo re tlo tsepelela diphihlelelo tša gagwe tše tharo tše kgolo bjalo ka go ba mmuši wa maemo, motsomi yo maatla le moagi. Nepo ya karolo ye ke go ganetšana le tshekatsheko ya go se be botse ye e nepilwego Nimirode nakong ya go feta. Re tlo tlaleletša ka go fa tshekatsheko ye e tebilego ya thogako godimo ga Hama/Kuše/Nimirode. Sa bohlano, ebile e le sa mafelelo, re tlo tšweletša nyanyeng bohlokwa bja kanegelo ya Nimirode go bao ba bego ba gateletšwe le go ba makgoba ka baka la temana ye. Re tlo nepiša Afrika-Borwa.

\section{Bana ba Noage}

Noage o be a na le barwa ba bararo, e lego Seme, Jafete le Hama. Nimirode ke setlogolo sa Hama. Hama e be e le morwa wa mafelelo wa Noage (9:24), gomme go tšewa gore Seme e be e le leitšibolo la Noage mola Jafete e be e le morwa wa gagwe wa bobedi (Mathews 2001:439; Jamieson et al, 1997). Thogako godimo ga Hama le moloko wa gagwe (go balwa le Nimirode) e hlatholotšwe gore e ra le thogako ya Bathobaso le meloko ya bona lehono. Tlhalošo ye e bile le ditlamorago tša go se kgahliše go Bathobaso.

Kanegelo ya thogako go Hama e ngwadilwe ka go Genesi 9:18-29. Noage o ile a robala a sa apara selo ka tenteng ya gagwe, ka lebaka la go tagwa ke morara wa go tšwa tšhemong ya gagwe. Hama 'o bone gore tatagwe o hlobotše a tšwela ka ntle go yo botša barwarragwe' (9.22). Seme le Jafete ba ile ba apeša Noage kobo ka go šia. Noage o ile a bolela thogako go morwa wa Hama, Kanana, mola a ile a šegofatša moloko wa Seme le wa Jafete (Willington 1997:13).

Go ya ka Gen. 10:21-32, Seme o tsebja e le tatagoBahebere bohle, ka lebaka la gore ditšhaba tše pedi di tšwele go Ebere ka Pelege le Jokotane, e lego ba leloko la Aborama, gape le setšhaba sa Baarabia ba leloko la Jokotane (dit. 26 go ya pele) (Keil \&Delitzsch 2002:107; Willington 1997:13; Hughes et al 2001:14; Mathews 2001:439). Bohlokwa bja leloko la Seme ke ge e le lešika leo Aborama le Kriste ka boyena, ba tšwago go lona (Richards 1991:31). Bao ba gopolelwa go 
ba batho ba setšo sa Semitiki go ya bohlabela bja Palesetina bao Bahebere ka bobona ba hlolegilego magareng ga bona (Gibson 2001:201). Moloko wa Jafete o tšweleletšwa mo go Gen.10:1-5. Mo temaneng ya 1, maina a barwa ba bararo a tšweletšwa go ya ka tatelano ya bogolo bja go tliša phethego le thumo go Tholedoth (Keil and Delitzsch 2002:102). Leloko la Jafete le amanywa kudu le dikarolo tša Palestina, e lego Asia Minor le Yuropa (Mathews 2001:431, 442; Gibson 2001:201; Keil \& Delitzsch 2002:104; Hughes et al 2001:14; Jamieson et al 1997). Go ya ka Genesi 9:18-27 bobedi Seme le Jafete ba be ba šegofaditšwe, mola Hama a be a rogakilwe go ba lekgoba la bona.

Bjalo ka ge re šetše re boletše, kanegelo ya bana ba Noage e laodiša thogako ye e beilwego godimo ga Hama (Kanana). Go laetšwa gore sebe sa Hama e bile sa go se laetše tlhompho go tatagwe, Noage. Basekaseki ba bangwe ba nganga ka go re thogako ya morwa wa Hama, Kanana, gabotse ke thogako go moloko ka moka wa Hama, go balwa le Nimirode, motlogolo wa gagwe (Hughes et al 2001:14). Banyakišiši ka kakaretšo ba dumelelana gore barwa ba Hama (10:6-20) ba ile ba hudugela seleteng sa borwa gomme go tšewa gore e be e le Maafrika le ditšhaba tša go fapana tša Babelone, Asiria le Palestina (Hughes et al 2001:14; Gibson 2001:201; Mathews 2001:431; Keil \& Delitzsch 2002:104). Keil le Delitzsch (2002:104) ba bolela tše di latelago malebana le tswalano ya moloko wa Hama le Maafrika:

Kuše: Baetopia ba bogologolo, bao ba sa dulego Afrika fela, eupša ba bego ba phatlalatše le borwa bja Asia ka moka, gomme mathomong, ka mo go laetšago, ba ile ba dula Arabia, fao ditšhaba tše di šetšego, di tswakatswakanego le Bašamaete di šomišago polelo ya Sešamaete. Mizraim ke Egepita: go laetša sebopego sa bobedi se ile sa tlošwa nageng go ya go batho, eupša re sa re go setereke sa bobedi, e lego, dikarolo tše pedi tša naga tšeo noka ya Naele e arolago naga ka gona eupša dinaga tše pedi tša Egepeta, ya Godimo le ya Fase, dikarolo tše pedi tša naga tšeo di fapanago kudu ka boso le tikologo ka bophara.

Leina la kgale la Seegipeta ke Kemi (Copt. Chêmi, Kême) leo Plutarch a rego le hlamilwe go mobu wa go teba wa go swana le molora wo o apešitšwego ke maraga a noka ya Naele; eupša go lego maleba kudu go o latišiša go Hama, le go laetša gore badudi ba yona ba pele e be e le Baitamitiki. Put o laetša Balipia ka kgopolo ya go phatlalala (Egipeta ya kgale: Phet; Copt. Phaiat), bao ba bego ba sabaletše le Leboa la Afrika go fihla ka Muritania, nakong ya Jerome (noka ye e bapile le tikologo ya boagišane, ye e bego e se na le leina la Phut) bona Bochart, Phal.iv.33 (Keil \& Delitzsch 2002:104).

Taba ye ya kamano magareng ga Hama/Nimirode le batho ba setlogo sa Afrika e tlo tšwetšwa pele ka botlalo ka morago mo taodišwaneng ye.

Go na le dihlopha tše nne magareng ga barwa ba Hama: Kuše, Mizraim (Egipeta), Put le Kanana. ${ }^{1}$ Ditšhaba tše šupa di bitšwa meloko ya Kuše (tem. 7) gomme tše šupa di tšwa go Mizraim (dit. 13 -14, go sa balwe "Bafilisita") (Mathews 2001:443; Jamieson et al. 1997; Carson 1994). Eupša go

1.Sarna o swaya gore tše tharo ke tša Afrika-Leboa, mola ya bone e le nagaletšibigo (Kanana) ye e kgokaganyago Afrika le Asia bohlokwa go swaya gore šedi ye kgolo ya mongwadi wa Genesi 10 ke Mohamaete "Nimirode", yo a tanyago šedi e le mmuši wa mathomo le moagi wa mafelo a ditoropo tša Mesopotamia (Mathews 2001:443).

\section{Seswantšho sa go befa sa Nimirode}

Go bohlokwa go lemoga gore go tšweletša seswantšho sa go befa sa Nimirode ga se taba ye mpsha. Banyakišiši ba lehono ba tšwetša pele kgopolo ye e thomilego mengwaga ye mentši ya go feta. Ditemaneng tše di latelago re tlo ahlaahla ka fao basekaseki ba go fapana ba tšweleditšego seswantšho sa go sekama sa Nimirode go tloga ka dinako tša kgale go swana le nakong ya borabi.

\section{Dikgopolo tša Bajuta}

Mo dikanegelong tša Bajuta Nimirode o tšewa e le mmolai wa go felafela dipelo. Kanegelo e laetša ka fao moloko wa barwa ba Noage o ilego wa kgetha barwa ba kgoši go ba buša ka morago ga Meetsefula. Ge Nimirode a kgethwa go ba morwa wa kgoši wa leloko la Hama, Jokotane o kgethilwe go ba wa leloko la Seme, gomme Pheneke a ba wa leloko la Jafete (Haynes 2002:42). Barwa ba kgoši ba bararo ba ba ile ba thoma go aga Mmoto wa Babele bjalo ka ge go laodišwa ka go Genesi 11. Nakong yeo 'banna ba go loka ba lesomepedi, ba magareng ga bona go bego go le Aborama, ba ile ba tlogela leano leo. Ge ba lesomepedi ba tlišwa pele ga barwa ba kgoši, Nimirode le Pheneke, ba ile ba befelwa kudu gomme ba tšea sephetho sa go ba lahlela ka mmolong' (Haynes 2002:42; Ginzberg 1968:174-80).

Kanegelo ye nngwe e bolela ka Nimirode ge a eba motho yo maatla. Nimirode o botha dira go tšwa molokong wa Seme le Hama gore go fedišwe moloko wa Jafete. Moloko wa Hama o bea Nimirode bogoši, gomme o fediša Basimete. Ge a šetše a na le maatla godimo ga meloko ya Noage, Nimirode o aga sebo se segolo godimo ga leswika la nkgokolo; a bea trone ye kgolo ya legong la cedar (seta) godimo ga yona go thekga trone ya bobedi, ye e bego e bopilwe ka tšhipi; ye e bego e thekgile terone ye kgolo ya koporo, trone ya silibere e le ka godimo ga ya koporo. Nimirode o hlomile trone ye botse ye kgolo, yeo a rego ge a be a dutše go yona maemong a bokgethegi, a swanelwe ke maemo le thompho ya legohle lohle (Haynes 2002:43).

Dikanegelo tša borabi le tšona di swantšha Nimirode bjalo ka motho wa go se tšhabe Modimo. O tšweletšwa e le motho yo a ilego a bopa le go hlankela medimo ya kota le ya leswika, a thekgwa ke morwa wa gagwe Maredone; gomme o ile a iša balatedi ba gagwe bobeng. Ditlamorago tša ditiro tša gagwe di laodišwa ka botlalo:

Batho ba ile ba se sa tshepa Modimo eupša ba tshepa maatla le bokgoni bja bona, e lego boitshwaro bjoo Nimirode a lekilego go retollela lefase ka moka go bjona. Ke ka fao batho ba ilego ba re, 'go tloga tholegong ya lefase ga a gona yo a swanago le Nimirode motsomi yo maatla wa batho le dibata, modiradibe pele ga Modimo'. Dikanegelong tše dingwe tša Nimirode, o rata go 'itšweletša e le modimo', gore ditšhaba ka moka di mo lobe.

(Haynes 2002:43) 
Borabi ba bone Mmoto wa Nimirode e le bogegelathoko kgahlanong le Modimo. Go ya ka kanegelo ye, Nimirode o ile a re:

Ke tlo ipušeletša go yena ka baka la go nweletša bagologolo ba ka. Ge a ka romela meetsefula gape, mmoto wa ka o tlo rotoga go feta le Ararata, gomme wa mpoloka.

(Haynes 2002:43)

Ge ba seno fetša go aga mmoto, baagi ba ile ba thuntšhetša mesebe godimo. Ge mesebe e boa go bona e kgamathetše madi, ba be ba goelela ba re: 'Re bolaile bohle ba ba lego legodimong' (Ginzberg 1968:179). Ge taba ya Babele e fetile, Nimirode o ile a tšwela pele go buša le go aga metsemegolo, ye a e tladitšego ka badudi, a ba bua ka seriti sa segoši (Haynes 2002:44; Ginzberg 1968:180). Borabi ba re botša gape ka tswalano ya Nimirode le borragoBajuta, kudu Aborama. Nonwaneng ye nngwe ya matswalo a Aborama, Nimirode ke radinaledi yo a laolago ka dinaledi gore ngwana o tla thenkgolla badimo ba a ba rapelago madulong a bona. Baeletši ba gagwe ba mo eletša go bolaya bana ka moka ba bašemane mo mmušong wa gagwe, e lego se a ilego a se dira. Ge ba bona polao yeo, barongwa ba ile ba llela go Modimo, 'Na ga se Wa šetša ka fao Nimirode moragaki a bolayago ba ba se nago molato ka gona?' Eupša Aborama yo a bego a se a hlwa a belegwa, ka mohlolo ga a lemogege popelong ya mmagwe. O a phologa, a tšwa mahlalagading ka matšatši a masomepedi gomme a ruta Nimirode ka semelo sa Modimo wa pale (Haynes 2002:44).

Go tšwa taodišong ye ya ka godimo, go tšwelela seswantšho se se latelago sa Nimirode:

(1) O bušitše barwa ba Hama morago ga meetsefula; (2) e bile mmuši wa lefase lohle, yo katlego ya gagwe e bego e amanywa le maleatlana; (3) o be a befelwa ka pela gape a na le boganka; (4) go no swana le barongwa bao ba welego, o be a laetša borabele le bogegelathoko kgahlanong le ba legodimong; (5) o laeditše lenyatšo ka go aga mmoto woo maekemišetšo, ka wona, e bego e le go phefa meetsefula a bobedi; (6) semelo sa gagwe sa go gana go bušwa le moya wa ntwa kgahlanong le Modimo, o bonagetše go baagi ba gagwe; (7) o hlohleleditše go ipota ga batho gomme a thoma taba ya go hlankela medimo le (8) go itira modigwana ka go gapeletša gore ditšhaba di mo lotšhe.

(Haynes 2002:45)

\section{Seabe sa Bokriste}

\section{BorragoKereke}

Bjalo ka borabi, bangwadi ba Bakriste ba nakong ya phatrisitiki ba gapeletšega go hlaloša taba ya Nimirode bjalo ka ge e loketšwe go Lenaneo la Ditšhaba mo go Genesi 10. Magareng ga botate bao, dingwalo tša Agostine ke tšona di bonalago di be di na le khuetšo ye kgolo. Augustine o lekile go hlaloša seswantšho sa motlogolo wa Hama ka go fetolela Genesi 10:9 '[Nimirode] e be e le motsomi yo maatla yo mogolo kgahlanong le Morena Modimo' (Augustine 1955:XWI:13). Phetolelo ya Augustine ya ka mo godimo e hueditše basekaseki/bafetoledi ba ba mo latetšego ka mekgwa ye mebedi. Sa mathomo e be e le kgopolo ya go re Nimirode o be a na le leemo le legolo. Magareng ga bangwadi ba Bakriste, kgopolo ye e ka latišišwa go Filaster ngwagakgolong wa bone le go Tertullian mo ngwagakgolong wa bobedi. Eupša mafelelong e theilwe methopong ya dingwalo tša Bajuta - e ka ba ka tsela ya 'Septuagint' ya Genesi (yeo e balegago ka go re 'Gomme Kuše a tswala Nimirode; ... E be e le motsomi yo mogolo yo maatla pele ga Morena Modimo) (Kugel 1997:128) goba 1Enoch, e lego puku ya apokhaleptiki go tšwa ngwagakgolong wa boraro B.C.E., (Kugel 1997:578) yeo e tšweletšago gore tswalano ye e bego e iletšwa magareng ga "barwedi ba batho" le "barwa ba Modimo' bjalo ka ge e ngwadilwe mo go Genesi 6, e tlišitše morafe wa makgema. Ka ge tlhalošo ya Nimirode mo go Genesi 10:8-9 e hlagiša lentšu la go swana le le tšwelelago ka go Genesi 6:4, la ('gibbor'; 'ramaatla') go haloša badudi ba lefase la 'prediluviun', babadi ba mathomo ba Bibele ba be ba tšea gore Nimirode o be a swanetše go ba e le yo maatla go ya ka lebopo la gagwe. Leemo la Nimirode e bile yona taba ye bohlokwa setšong sa go hlatholla ge e be e thekgwa ke Augustine (Haynes 2002:46).

Khuetšo ya bobedi mo go basekaseki/bafetoledi ba bangwe e be e le maemoa Augustine a go re Nimirode e be e le motsomi kgahlanong le, go feta go ba motsomi pele ga Morena. Augustine o feditše ka go re leina la motsomi le 'ka šišinya fela moradia, mogateledi le mofediši wa dibopiwa tša lefaseng' (Augustine 1955:XWI:13). Ka kopanyo ya phetolelo le tlhathollo, Augustine o tšweleditše Nimirode e le lenaba la Modimo le molwantšhi wa boikokobetšo bja kgonthe. 'Tselakgolo ye e bolokegilego ya go botega ya go ya legodimong, e agilwe ke boikokobetšo ye e hlatlošetšago pelo ya yona go Morena, e sego kgahlanog le Morena, bjalo ka ge go dirile lekgema leo ...', gwa realo Augustine (Augustine 1955:XWI:13). Go ka thwe go ukama gore Nimirode o be a ganana le Modimo go hlalošwa ka tshwanelo kgopolong ya Augustine ya go tšea gore yena, 'le balatedi ba gagwe, ba ile ba thoma go aga mmoto kgahlanong le Modimo, e lego seo se bego se laetša boikgogomošo bja go tlala lenyatšo' (Haynes 2002:46; Augustine 1955:XWI:13).

Bangwadi ba mmalwa ba 'pharasitiki' ba šitletše tswalano ye e sego ya Nimirode le bosehlogo. Go fa mohlala, Jerome (347-420) o gatelela gore "Nimirode, morwa wa Kuše e bile wa mathomo wa go tšea maatla ka kgang le ka bosehlogo [ao pele] a bego a sa ka a šomišwa, godimo ga batho (Haynes 2002:47). Sengwalong sa gagwe sa Recognitions, Clement o katološa sebopego se sa Nimirode le leloko la gagwe ka tsela ye:

Molokong wa bolesomešupa Nimirode wa 1 o ile a buša Babelone, gomme a aga toropokgolo, a efa Baperesi, le go ba ruta go rapela mollo. Molokong wa bolesomesenyane, moloko wa yoo a bego a rogakilwe ka morago ga meetsefula [Hama], ge ba be ba tshela mellwane ya maleba ye ba bego ba e humane ka matengwa dikarolong tša ka bodikela, ba rakela bao ba bego ba humane karolo ya gare ya lefase thoko ya bohlabela... mola bona ba thopile naga yeo ba rakilego [badudi ba yona] ka boganka.

(Haynes 2002:47)

Go Clement, Nimirode o tswalanywa le go aga toropo le go hlankela medimo, gape ba moloko wa gagwe go thwe ba ekiša maitshwaro a kgatelelo ao a tlogo tsebja ka ona. 
Clement o swaya Nimirode phošo ka go thulana le leano la Modimo la go gašana ga batho ka morago ga meetsefula; tsholo yeo e tlilego ya ba tabakgolo historing ya thathollo ya Bibele (Haynes 2002:47).

Basekaseki ba 'pharasitiki' ba rwešitše Nimirode bomohola bja sekao le bja histori. Seabe sa gagwe mo dikanegelong bjalo ka motsomi, moagi wa mmoto, le mogateledi, se mo dirile seka sa maleba sa boikgogomošo bja motho le bogogelathoko. Ge nako e sepela, Nimirode o ile a tla a emela taba ya 'go gomarela dilo tša lefase kudu; maikemišetšo a mabotse eupša a go hloka tsela ka ge nepo ya ona e be e se ya lebantšhwa go Modimo eupša go dithoto tša mo lefaseng (Haynes 2002:48).

Ka lehlakoreng le lengwe, le ge dikanegelo di tswalanya Nimirode le Babelone, lešika la Nimirode go tloga go Hama le Kuše le dirile gore bangwadi ba ditaba tša borragokereke ba mo tšee bjalo ka Moafrika. Ba bangwe ba bolela gore 'ka Sehebere "Chus" e ra 'Aethiops'; ba bangwe ba re 'Nembroth e ra Aethiops.' Ditabeng tše ka moka, Nimirode le mmoto wa gagwe di dirilwe tša Afrika ka lebaka la tswalano ya gagwe le Hama. Kopanyo ya dika tša semoya le tša tatelano ya meloko tšeo ka setlwaedi, di amanywago le Nimirode, di dirile gore Ambrose a fetše ka go re e be e le moemedi wa lehlakore la lewiswi la botho:

A gapeletšwa ke thago ya gagwe ya go phela le go dira dilo bjalo ka phoofolo go feta sebopiwa sa go inaganela, Nimirode ke seswantšho sa moya wa molato, 'Moitopia, lenaba la seetša, yo go sego kganya mo go yena'.

(Haynes 2002:48)

\section{Mehlagare}

Kanegelo ya Nimirode e ile ya tšwela pele go anegwa nakong ya Mehlagare ya Sekriste. Nimirode o ile a tšwela pele go amanywa le tsebo ye e utamilego; a rwešwa diala ka tšohle go tloga ka go hlama profeto go tsebiša ba Boblale ka Matswalo a Jesu, go fihla ka go utolla mehlolo ya dinaledi, go ya go tsebo ya bobetli (Haynes 2002:48).

Ye nngwe ya ditšo tša nako ya kgale e be e amantšha Nimirode le morwa wa semaka wa bone wa Noage. Go be go bolelwa gore morwa wa bone wa Noage, Yonthone, (Jonathane), o sepetše go ya Bohlabela, a kopana le Nimirode (Gero 1980:321-30). Gona fao Jonathane o ile a ruta kgošikgolo ye bohlale bja polelo - e lego thuto ya paale ya dinaledi. Go bolelwa gape gore Nimirode le yena e be e le morutiši. 'Kutollo ya Nimirode' e be e gopolelwa go ba profeto ya Sekriste ya go tla ga Mesia, yeo tsebo ya yona e tlišitšego ba Bohlale ba Bohlabela Betlehema (Haynes 2002:48; Gero 1980:321-30).

Ka ngwagakgolo wa boselela, Nimirode e be e setše e le radinaledi, 'gomme ya dula e le radinaledi magareng ga banna ba Mehlagare'. Sengwalo se sengwe sa dinako tša kgale se na le sethalwa sa Atlas le Nimirode, magoši a mabedi 'ao ditšo tša klasike le bogologolo di ba dirago bathomi ba thuto ya boradinaledi.' Go ya ka sengwalwa se, 'Atlas o tšweletšwa a eme godimo ga Pyrenees gomme godimo ga gagwe go le leratadima le dinaledi, mola Nimirode a eme godimo ga thaba ya Baamorate gomme a lebeletše godimo ka go thekga magodimo a a se nago le dinaledi matsogong a gagwe (Haynes 2002:49).

Mahlakore a kanegelo ye a tšweletšwa pele ya Nimirode ke Alighieri Dante ka sebopego sa gagwe se segologolo, kgatelelo ya gagwe go batho, le maikarabelo a gagwe mo tharanong ya maleme a Babele. Dante o thopolla Nimirode tikologong ya dihele fao go nago le makgema a dinonwane tša kakanyatlhalošo le dikanegelo; gomme o mo hlaloša ka botlalo maemong a bobe (Haynes 2002:49).

\section{Mpshafatšo le Tsošološo}

Mo go Lectures on Genesis, Martin Luther o laetša ka fao kakanyo ya Sekriste e tswalanyago Hama le Nimirode kgauswi le kgauswi ka gona. Ga se fela gore baanegwa bao ba na le lapa la go swana, eupša ba thoma go ba selo se tee ge Luther a nea Hama dika tše bohlokwa tša kanegelo ya Nimirode (Haynes 2002:50). Mohlala, o bea Hama Babelone, fao 'mmogo le meloko ya gagwe, a swaraganago le go aga toropokgolo le mmoto' (Luther 1955:IX,175). Eupša Luther ga a beele Nimirode thoko, yoo, 'ka morago ga go humana maatla ka kgatelelo, a hlorišago meloko ye mekgethwa ya Noage ka ditsela tša go fapana, le ka go ithomela mmušo, gomme ya ba yena mmušimogolo a nnoši godimo ga wona' (Luther 1955:210). Setlogolo se swana le rakgolo 'a sona: Bjalo ka ge Hama a nyaditše tumelo le setšo sa Noage ka go nyefola tatagwe le go hloma mmušo wo mofsa le tumelo ye mpsha, Nimirode le yena 'o senyeditše mmušo le kereke. Ga se a hlohleletša bodumedi bja nnete' (Haynes 2002:50).

Go latela borragokereke, Luther o latofatša Nimirode ka go tliša 'kgatelelo godimo ga batswala ba gagwe, bao a ilego a ba raka dinageng tša botatagomogolo ba bona' (Luther 1955:212). Taba ya thwi ke gore, Bahamorate ka tlase ga Nimirode ba thopa karolo ya naga ye e bego e filwe ba Seme, 'mojabohwa wa tshepišo ya Kriste' Ka lebaka la maitshwaro a Bahamate ba tlokwa ka polelo ya setimone:

Le ge go se na bohlatse bjo bo ngwadilwego bja seo ba lekilego go se dira kgahlanong le kereke ya nnete, le kgahlanong le Noage ka boyena e lego molaodi wa kereke, gape le kgahlanong le leloko la gagwe la ka moso le a ikgantšhago ka lona, eupša go ka se phopholotšwe ka go bapetša ge re hlokomedišiša ditiro tša baganetši ba rena nakong ya bjale, ka gobane Sathane, yo a hlohleletšago ba babe kgahlanong le kereke ya paale, ga a fetoge.

(Luther 1955:219)

Bjalo ka lenaba la Noage le meloko ya gagwe ya ka moso ye a ikgantšhago ka yona, Nimirode o ipakela go swantšhwa le yo mobe (Haynes 2002:51).

\section{Mengwagakgolong ya Lesomeseswai le Lesomesenyane}

Nakongyamegwagakgoloyalesomeseswailelesomesenyane, dirutegi di be di se na le dikakanyo tše di šetšego di le gona ka Nimirode, tšeo di balago gore: Nimirode ke mohlala wa mogateledi wa kgale yo a hlotšego go rapela medimo ka go aga toropokgolo le mmoto, yeo a beakantšego mmušo wo mogolo go e dikologa (Haynes 2002:57). 
Matthew Henry (Matthew 1996:76-77) o tšweleditše tlhalošo ya go fetiša ya go hlatholla ka Nimirode. Go ya ka Henry, sa mathomo Nimirode e be e le moamogi: 'Nimirode e be e le mothopi ka dintwa wa ditokelo le dithoto tša baagišane ba gagwe, gape e le mohloriši wa banna ba ba se nago molato; a rwele tšohle pele ga gagwe, a ikemišeditše gore tšohle e be tša gagwe ka kgapeletšo le boganka'. Sa bobedi, o be a hlankela medimo e šele. Go swana le Jereboamo, Nimirode o ile a tliša go hlankela medimo e šele e le go tiiša maatla a gagwe. 'Gore a tliše mmušo wo mofsa, o ile a hlama tumelo ye mpsha godimo ga go fediša tša kgale tše pedi.' Sa boraro, e be e le motsomi kgahlanong le Morena: 'O tšwetše pele ka kgatelelo le boganka a nyatša Modimo... tše nkego yena le batsomi ba gagwe ba be ba le bogale go feta Ramaatlaohle. Sa bone, e be e le radipolitiki yo maatla.' O beile motheo wa bogošikgolo, bjoo ka morago e bilego hlogo ya gauta, le boganka bja ba maatla, a le yo botse go legohle... Bona botala bja mmušo wa batho, kudu sebopego sa wona se se beago maatla a magolo go motho o tee'. Sa bohlano, e be e le moagi wa go makatša. Go bonala Nimirode a ka be e le yena mohlami wa Babele. Ge morero wa gagwe wa go buša leloko la Noage o phuhlama ka tšharakano ya maleme, Nimirode o ile a sepela a yo aga Ninife. Sa boselela, e be e le thuto ya kgonthe ka dikganyogo tša botho: Go Nimirode re ithuta gore kganyogo ga e na mellwane, ga e khutše, e a bitša, gape e na le ditlhohlo. Sa mafelelo ke gore o be a tšewa go ba legogelathoko: 'Leina la Nimirode le emela borabele, bjoo (ge e ba ka nnete o šomišitše maatla a gagwe bošaedi, go gatelela baagišane ba gagwe) bo re rutago gore banna ba bagateledi ke balwantšhi ba Modimo, gomme go se kwe ga bona go swana le sebe sa boloi' (Matthew 2001:76-77; Haynes 2002:57).

Tlhathollo ye ya go sekama ya ka godimo ya (Hama) Nimirode e ile ya tšwetšwa pele ya bewa godimo ga Bathobaso. Go be go ngangwa ka la go re Nimirode e be e le yo moso ka mabaka a mararo. Sa pele, rakgolo wa gagwe Hama ke Moafrika wa mathomo. Sa bobedi, tatagwe Kuše ke mogologolo wa Baitopia. Sa boraro, o emela lehlakore la leswiswi la thago ya batho. (Haynes 2002:60-1). Ka fao, Bathobaso ka moka ba be ba bonwa e le moloko wa Hama/Nimirode ke ka fao le bona ba rogakilwe. Tlhathollo ya mohuta wo e hlotše tshenyo ye kgolo bophelong bja motho yo moso. Go latela tshwayotshwayo ya tšhomišo ya kanegelo ye go gatelela Bathobaso.

\section{Tšhomišo ya kanegelo ya Hama/ Nimirode kgatelelong/ bokgobeng bja Bathobaso \\ Ka fao temana e dirišitšwego ka gona}

Genesi 9:18-27 e šomišitšwe go thekga kgatelelo ya Bathobaso mo Afrika-Borwa, le Dinagengkopano tša Amerika ka go nganga gore ba rogakilwe go tloga dinakong tša Bibele.

Go ya ka kgopolo ye, Bathobaso ba na le 'thogako' ya Hama (gabotse, thogako ya Kanana, e lego ngwana wa Hama) ye e lego godimo ga bona ka baka la sebe sa Hama go Noage, seo
Noage a ilego a mo rogaka ka lebaka la sona (Gen. 9:25-27) (Williams 2004).

Mengwageng ya pejana, James W.C. Pennington, o ile a tsošološa taba ya Nimirode go hlaloša histori ya go nyefolwa ga Maafrika. Le ge Pennington a be a eme ka la go re Bathobaso ke moloko wa Kuše le Mizraim, o ile a kwa a gapeletšega go hlaloša gore ke ka lebaka lang Maafrika a ile a tlogelana kudu le bodumedi bja go hlweka bja Noage. Go ya ka Pennington, mongmolato e be e le 'tumelo ye e hlamilwego ke Nimirode, [yeo] e ilego ya ipha maatla ka moragonyana ga lehu la gagwe, ka ge a be a khunamelwa ke ba leloko la gagwe. Ke Beluse goba Baale go histori ye kgethwa. Baitopia ba moloko wa bobedi ba ile ba šala tumelo ye morago, gomme ya tsenywa mo go thutatumelo go mmušo, le dingwalo tša bona' (Pennington 1841:32; Haynes 2002:111). Pennington o humane kanegelo ya Nimirode e šomišega go hlaloša bootswa, bohetene, go hlankela medimo e šele, le go phasa badimo moo go bego go le gona Afrika nakong ya gagwe (Pennington 1841:32; Haynes 2002:111).

Sandell (1907), yo a ngwadilego mengwaga ye mentši ka morago ga Pennington o ile a hlatholla thogako ya Noage go ra go re morafe wa Maafrika o be 'o profetetšwe go ba maemong a tlase go na le a Seme le Jafete, ebile o be o se wa swanela go ka ba le mmušo wa wona, gape o be o sa swanela go laola ka kerekeng goba mmušong (Sandell 1907:41). Go no swana le 'Ariel', Sandell le yena o šomiša kanegelo ya Nimirode, le ge yena a tsepeletše godimo ga maatla a yona bjalo ka motheo wa kgethollo ya semorafe. 'Ke thogako go tlhago go nyaka go gapeletša go lekanya merafe ya go fapana kudu', gwa ngwalwa bjalo Sandell. Se se laeditšwe ke maitekelo a bobe go nyaka go aga Mmoto wa Babele, woo e lego seka sa 'kganyogo ya go nyaka go ba ba bagolo le go ba le leina bjalo ka setšhaba se tee se segolo.' Gobane 'tumo ya mmušo o tee wo mogolo wa go ba le motho o tee e le yena mmuši wo mogolo, ga e thabiše pele ga Mmuši yo Mogologolo wa legohle... Modimo o ile a aroganya merafe ya batho gomme e phatlaladitšwe le lefase ka moka'(Sandell 1907:48). Go ya ka Sandell, mmoto ke seka se Batlemaganyi ba go wa le go tsoga ba se tšeago e le tšhošetšo ye kgolo go tokologo ya batho - mmušo wa bogare wa maatla, wa bofeterasi. Sandell o bapeditše mebušo ye bjalo le wa Babele (Gen. 11) le Babelone ka go swana (Haynes 2002:113).

Gabotse, bathekgi ba botee bja tumelo bao ba ngwadilego seripeng sa bobedi sa bo-1950, ba hlathile Genesi 9:20-27 bjalo ka motheo wa maikutlo a karogano. Bathekgi ba thogako ya Noage ba hlatholotše thogako go ba Monegro, bjalo ka moloko wa go tšwa go Hama, wo o hlomilwego ke Modimo go ba maemong a fase a go ya go ile mo setšhabeng, le gore a ka se tsoge a tšerwe go ka lekana le Mothomošweu. Go ya ka thogako ye, ba bangwe ebile ba tšea gore Monegro o maemong a tlase go tloga tswalong, ka fao a ka se kgone go ikemiša goba go hlatlošetšwa maemong a godimo a ditšhaba tše dingwe ka mogopolo, setšo le ge e ka ba ka maitshwaro (Haynes 2002:116).

Gillespie (1954) yo a ilego a nyakolla metheo ya semorafe go ya ka Bibele, o ile a gatelela kgopolo ye e ilego ya tumišwa 
ngwagakgolong wa bolesomeseswai mererong ya semorafe gore ka morago ga Meetsefula, barwa ba bararo ba Noage e bile botatagomerafe ye meraro ya go fapana, yeo e bego e swanetše go atiša batho le go ba phatlalatša le lefase ka moka. Go ya ka Gillespie, meloko ya Seme e ile ya dula bogolong bja Asia, meloko ya Jafete e ile ya leba go la Yuropa, mola bana ba Hama ba lebile borwa lehlakoreng la melatšatši le kontinenteng ya Afrika (Haynes 2002:117-118).

Go ya ka Gillespie (Gillespie 1954:4,11), go šarakana ga maleme Babele le go phatlalala ga batho mo go latetšego, e be e le 'tiro ya go ikgetha ya go tšwa go Modimo go šarakanya maikemišetšo a batho ba kgopo go kgonthiša go tswakatswakana ga batho ba lefase go ya go ile.' O ile a gatelela gape gore go tšwelela ga maleme a go fapana e bile taba ya maleba go bona gore merafe ya go fapana e dule e kgaogane. Ditlhathollo tša mohuta wo le ditemana tše dingwe tša Bibele di bopa kgwekgwe ya dikgopolo tša Gillespie tša go re kgethollo ya semorafe e sepetšana le Sekriste (Gillespie 1954:4,11; Haynes 2002:117).

Ka ngwaga wa 1956, Kenneth R. Kinney o tšweleleditše kgopolo ye bjalo ka kgethologanyo ya semorafe (Kinney 1956:9-10; cf Haynes 2002:117-118). Kinney o lla ka la go re, 'e be e ka se tsoge e bonwe ka lebaka la maitshwaro a go hlakahlakana a lapa la Hama' (1956:9-10). Kgethologanyo go ya ka semorafe ke tharollo ya Kinney go kgonthišiša maemo a ditaba a mabotse a ka moso. Gabotse, ka ge Modimo a nyakile gore dihlopha tša pele tše tharo di arogane go ya ka malapa le ditšhaba, meloko ya dihlopha tše e tlangwa go ya ka mangwalo gore le yona e latele tsela yeo. Gape manyalo a go tswakana magareng ga dihlopha tša Jafete (Mauropa), Seme (ba Bohlabela/Maašia) le Hama (Maafrika) a swanetše go thibelwa. Go swana le Gillespie, Kinney ga a bolele Nimirode ka leina. Eupša o šupetša go Genesi 10:6-9 le 11:19, e lego tšona ditemana tšeo babadi ba peleng ba Bibele ba di šomišitšego go amanya Nimirode le borabele bja Babele! Bangwadi ba ba laetša gore nakong ya sephetho sa ka bjako sa Kgorokgolo ya Tsheko sa go fediša 'kgethologano gore go be tekatekano' dikolong tša mmušo, makgethepolelo a borabele le go hlakahlakana ga maemo a dilo a dikile a tšweletšwa ka maatla ge go balwa Genesi 9-11 mabapi le kgethologanyo ya semorafe ngwagakgolong wa bolesomeseswai, gomme taba yeo e ile ya tsoša gape dikgopolo tša tumelo ya bomaganaphetogo (Kinney 1956:9-10; Haynes 2002:118).

Ka Afrika-Borwa, Genesi 9:18-27 e ile ya šomišwa go bapela le Joshua 9:27 ye e bolelago ka fao Joshua a ilego a dira ba Gibione 'batopeledi ba dikgong le bageledi ba meetse' go thekga taba ya go beela ba ba itšego mešomo le gore ka setlogo Bathobaso ba be ba lebanwe ke mešomo ya matsogo (Wittenberg 1991:46; Gibson 2001:201).

\section{Tshekatsheko ya thutatumelo ya thogako godimo ga Maafrika}

Le ge thogako ya Hama, yeo e rago le setlogolo sa Nimirode e šomišitšwe thwi go gatelela Bathobaso ba lehono ka AfrikaBorwa go akaretšwa le Amerika, go na le ditaba tše mmalwa go tšwa dikanegelong tša Hama tše di hlokomologilwego ke batho ba ba go šomiša ditemana tša Bibele ka bošaedi.

Go ya ka maemo a serafe, go na le diphošo tše mmalwa mo go peakanyo ka dihlopha kanegelong ye. Sa mathomo, Genesi 10 e bolela Elam e le morwa wa Seme. Eupša Baelamo, ba ba lego Iran goba Persia/Peresi lehono, ga se Basimate ka morafe (Gibson 2001:201).

'Elamo' ga se morafe wa Basimitiki eupša go thwe ke morwa wa Seme (Tem. 22) (Mathews 2001:434). Sa bobedi, ga go na go swana mo tabeng ya barwa ba bararo ba Noage. Ka go 9:18 barwa bao ba balwa e le Seme, Hama le Jafete. Ke tatelano ye bjalo le ka go Genesi 10. Eupša kanegelo ya thogako ya Noage e bonala e šišinya gore Kanana ke yo mongwe wa barwa ba Noage, ka ge mo go 9:25 Seme le Jafete ba bitšwa barwarragwe. Bothata bjo bongwe ke gore temana ya 24 e bolela ka Kanana e le morwa wa mafelelo go barwa ba Noage. Go molaleng gore se se thulana le lešika fao Hama, yo go temana ya 22 a bitšwago tatagoKanana, e lego morwa wa bobedi.

Bothata bjo bongwe ke bja fao thogako e lebantšwego gona. Dithogako le ditšhegofatšo mo ditemaneng tša 25-27 di lebišitšwe go Kanana, eupša go ya ka temana 22 ke Hama tatagoKanana yo a dirilego taba ya go hlabiša dihlong. Potšišo ye boradithuto bao ba thekgilego kgethollo le kgatelelo ya Bathobaso go ya ka thogako ya Noage ba šitwago go e botšiša ke ye: Ke ka lebaka la eng bobedi morwa le setlogolo (Nimirode) ba rogakwa ka seo se dirilwego ke tatabo? Ka lehlakore le lengwe, ge Kanana e be e le modiri wa taba ye ya mahlabišadihlong, ke ka lebaka lang go be go swanetše go rogakwa tatagwe? (Wittenberg 1991:48). Sa boraro, magareng ga bana ba Kuše, go na le tlhopho ya go ba pepeneng ya Bakanana le ditšhaba tša Afrika: Eupša, Bakanana e be e le Basimatiki bjalo ka Bahebere, ba dula nageng e tee ba bolela le mmolelo o tee (Gibson 2001:201; Mathews 2001:434). Sa bone, go na le dipoeletšo mo kanegelong (Gen. 10). 'Havilah' le 'Sheba', go fa mohlala, ba tšwelelala go bobedi dihlopheng tša Bahamite le Basemite (ditem.7 le 28-29) (Mathews 2001:434). Go filwe ditšhišinyo tše dingwe eupša ka bophara tša se amogelwe. ${ }^{2}$ Go lekane go no swaya ntlheng ya bjale gore peakanyo ke ya semorafe le tikologo, bjalo ka ge go laetšwa ka kgatelelo ya go ipoeletša ya mellwane ya dihlopha tša 'ditšhaba' (ditem. 5, 19, 30, 32) e sego ka maleme goba semorafe (Mathews 2001:434). Gunther Wittenberg (1991) o bolela gore meloko ya Seme, Hama le Jafete ga e emele dihlopha tša semorafe goba mehlobo, eupša peakanyo ya leago le sepolitiki.

Sa bohlano, banyakišiši ba bangwe ba tšea taodišo ya thogako ya Hama e le tlhalošo ya tlholego ya bonaba magareng ga Baisraele le Bakanana. Gibson o re:

Mo e tloga e le mothopo wa thogako ya Kanana mo kanegelong. E laetša lehloyo la go tsenelela le le lego mo dintweng tša go

2.Oded (1986:31) o šišinya gore mathomong dikarolo tše tše tharo di be di emela ditšhabana tše tharo: boralewatle (Jafete); ditšhabana tša go se dule felo gotee (Seme) le ditšhabana tša balemi tša sekgoweng (Hama); eupša ka morago gwa ba le (Seme) le ditšhabana tša balemi tša sekgoweng (Hama); eupša ka morago gwa ba le diphetogo tše di sa sepedišanego le maikemišetšo a mathomo a mongwadi, ka fao
tša šira peakanyo ya motheo le go thibela tlhathollo ya rena ya maleba ya temana ye. 
thopa, gape e laetša tšhošetšo yeo tumelo ya Bakanana ya go gatelela tša thobalano e bego e na le yona go hlweko ya tumelo ya Baisraele.

(Gibson 2001:201)

Ntlha ya boselela, ye e sa thewago godimo ga dihlopha tša semorafe ke ya gore, go ya ka temana (Gen. 9:18-27), Noage ga a rwešwe molato ka ditiro tša gagwe tšeo di bakilego thogako ya Hama. Ka lebaka la tiragalo ye ya go hlabiša dihlong, ga go tsholo yeo e pharwago Noage, eupša tsholo e fetišetšwa go Hama. Baradithuto ba ga se ba ka ba botšiša potšišo ye: Ke ka lebaka lang šedi ya tsholo ka moka temaneng ye e lebanywa le ditiro tša Hama e sego Noage, yoo ka maitshwaro a gagwe a go nyamiša a beilego morwa wa gagwe yo monnyane (9:24) tebanong le maitshwaro a tatagwe a go tšhoša, yo a bego a swanetše go ba mohlala go yena?

Go tšwa ditherišanong tša ka mo godimo, go laetša gore ga go ka fao motho a ka laetšago toka ntle le pelaelo ya kgatelelo ya Bathobaso ka gore Genesi 9 le 10 di a e thekga. Polelo ya ka godimo e laeditše gore go na le mathata a dingwalo, a bobedi Genesi 9:18-29 le Genesi 10 a lebanego le dithulano le go se eme felo gotee ga dingwalo tšeo. Gunther Wittenberg (1991:51) o laeditše ka mo go kgodišago gore peakanyo ya ditšhaba tša go fapana ke tlhopho ya maitirelo go ya ka tsela ya lešika ye e sa laetšego kgonthe nakong efe kapa efe historing:

Lešika la Noage le dira tše nkego le akaretša lefase ka moka la pele ga Israele le setšhaba sa lona, eupša kgabagareng yeo, mmepe wa phatlalalo ya ditšhaba wo o tšwelelago go palo ya dinaga le ditšhaba o ka se amanywe le nako ye itšego mo historing.

(Gibson 2001:201)

Ge nkabe bohle ba ba tšweleditšego seswantšho sa go befa sa Nimirode, ba theile dikgopolo tša bona godimo ga thogako ya Noage, ba ile ba šetša ka tsinkelo tše dingwe tša dintlha tše di ukamilwego ka mo godimo, nkabe ruri ba ile ba lekodišiša ditlhathollo tša bona.

Bjalo ka ge re sekasekile ka fao Nimirode a tšweleditšwego ka seswantšho sa go befa ka gona dingangišanong tša boradithuto ka gona, re tlo tšwela pele, mo karolong ye e latelago, go lekodišiša dikatlego tša gagwe e le go mo fa tshekatsheko ya mmakgonthe.

\section{Re Lebeledišiša Dikatlego tša Nimirode}

Karolong ye re ahlaahla dikatlego tše tharo tše kgolo tša Nimirode e le mmuši yo mogolo, motsomi le moagi yo maatla, e le go ganetšana le thathollo ya go befa ka banyakišiši ba bangwe.

Sa pele, re ahlaahla taba ya Nimirode bjalo ka mmuši yo mogolo yo a theilego Babilonia. Ga go pelaelo ya gore Nimirode e bile wa mathomo go ba 'yo maatla lefaseng.' Babele ke mathomo a mmušo wa gagwe, wo o ilego wa tšwela pele go gola (Gen. 10:8-10). 'Naga ya Nimirode' (Mika 5:6) ke tšhupo go Asiria goba ya Sinare, ye e lego karolo ya yona
(Witenberg 1991:51). Nimirode e be e le mmuši yo maatla (tem 10). O ile a ba yo maatla; ka fao a bea metheo ya bogoši, bjoo ka morago e bego e le hlogo ya gauta, le letšhogo/poifo go ba maatla, e le bokgabo go bohle le tšohle. Ga go laetše gore o be a na le maloka a go buša ka matswalo a gagwe; eupša e ka ba maemo a gagwe a go swanelwa ke pušo, a a mo filego sebaka go ka hlaolwa, bjalo ka ge ba bangwe ba akanya, goba ka maatla le maikemišetšo o tšwelełetše a eya pele go tšea mphapahlogo (Easton 1996; Walvoor et al 1985:43; Keil \& Delitzsch 2002:448).

Sa bobedi, re ahlaahla Nimirode e le motsomi yo maatla. Kanegelo ya Nimirode e re botša gape gore e be e le yena motsomi wa thwadi yo a bego a tsebega (ditem 9-10). O tšweletšwa e le motsomi yo maatla, e lego leswao leo le humanwago gantši go magoši a Asiria (Matthew 2001:76).

Tswalanyo ya 'mohlabani yo maatla' (tem. 8) le bogale bja motsomi ('motsomi yo maatla', tem. 9) e laetša gape ditšo tša mengwageng ya pele tša magoši a Egipeta le Mesopotamia ao a bego a tsebja ka tiro ye. Ditlhathollong ka bobedi 'gibbor' ('maatla') e šupago maatla a Nimirode e le mohlabani wa thwadi, motho yo a itirelago leina ka ditiro tše bogale tša go tšhabega. Gantši lentšu le le tšwelela ge go bolelwa ka dikatlego tša bohlabani (mohl. Joshua 10:2; Amosi 2:14; 16) (Walvoord et al. 1985:48). Baradithuto ba bangwe ba hlatholotše seema sa go re: Go swana le Nimirode, motsomi yo maatla pele ga MORENA' go ra gore Modimo o be a sa kwane le mabokgoni a Nimirode a go tsoma. Borathuto ba bangwe ba re sekafoko sa 'pele ga Morena' ga se šišinye gore Modimo o be a dumelela se Nimirode a se dirilego, eupša gore ke ka tlhokomelo ya Gagwe go batho ge a mo dumeletše go dira bjalo (Mathews 2001:448; Mathews 2001:448; Keil \& Delitzsch 2002:104-5). Eupša, kgopolo ya ka ke ya go re bokaone ke ge e ka tšewa e le mabobo a Modimo go Nimirode, ka ge go se na tšhupetšo go tšwa mo temaneng gore ka nnete Modimo o be a sa kgahlwe ke mabokgoni a Nimirode a go tsoma.

Sa mafelelo re ahlaahla Nimirode bjalo ka moagi yo mogolo. Nimirode wa ka Bibeleng ke kgoši ya mathomo ye maatla lefaseng; ditoropo tša mathomo tša mmušo wa gagwe e be e le Babelone ye e tsebegago kudu, Erech le Accad mo Babilonia, Ninife le Calah mo Asaria (tem.10). E be e le motsomi yo maatla 'ka kgaugelo ya Morena'. Nimirode bjale e be e tlo ba mogalemothei wa mebušo ye maatla ya Bohlabela yeo e bego e tšhošetša Baisraele nakong ya yona. (Gibson 2001:203; Clifford 1985).

Nimirode e be e le moagi wa go tsebega. Go a kgonega gore e be e le moatšhitheke ge go be go agwa Babele, fao a hlomilego mmušo wa gagwe (Clifford 1985; Walvoord et al 1985:43). Go latela thalošo ya Nimirode bjalo ka mohlabani. Go begwa gape le ka bogolo bja mmušo wa gagwe (10:10 12). Go bonagala go na le magato a mabedi, la pele ditoropo 'mo Shinar' (tem. 10) (Mathews 2001:450). Lentšu la Sehebere 'rešit', leo ge le hlathollwa e lego 'mafelo a mathomo,' a ka bitšwa gape 'mafelo a magolo,' go laetša bogolo, e sego fela 
tatelano. Ditoropo tšeo di laetšwago go ba 'nageng ya Šinare' di beilwe gotee (tem.10). 'Šinare', goba 'naga ya Šinare' (11:2, 'Babilonia', Daniele 1:2; Sakaria 5:11), di bala gabotse selete sa Babilonia seo bogologolo se tsebjago e le 'Sumere le Akada' (Matthew 2001:76).

Go tloga Šinare Nimirode o ile a ya Asiria, naga ya Bohlabela bja Thikirisi, gomme fao a aga ditoropo tše nne, goba gabotse motse wo mogolo wa borena wo o bego o na le ditoropo tše nne, goba motse wo mogolo wa borena wa ditoropo tše di filwego maina (Mathews 2001:450). Ka fao Genesi 10:11 e hlaloša kgolo ya khuetšo ya Nimirode seleteng sa Asiria (Sehebere aššur; Akk aššur). Popafoko ye e emetšwego e tlo bala Asshur e le kgokasediri; ka fao, 'Asshur o ile a tšwa a yo aga,' eupša popafoko e dumelela Nimirode e le kgokasediri ('o') ye e tswalanago gabotse le moela wa tlhalošo ya kanegelo fao Nimirode a sepelago 'go tloga nageng yeo', e lego Šinare, go ya tikologong ye mpsha ya Asiria. Tlwaelo ye e tšweletšwa ngwagakgolong wa lesomeseswai ke moprofeta Mika, yo a bolelago ka 'naga ya Nimirode' (5:6; Keil \& Delitzsch 2002:105-6; Mathews 2001:450).

Ninife e tšwetšwa pele e le toropo ya maemo seleteng seo. E ikadile leribeng la ka bohlabela la karolo ya ka godimo ya Noka ya Tikirisi go lebana le toropo ya Mosulu (leboa la Iraka) gomme e na le histori ya go ya dinakong tša mathomothomo. Ka moragorago e ile ya ba bogare bja mmušo wo mofsa wa Asiria mengwagakgolong ya boseswai le wa bošupa wo o bilego le khuetšo ye bohloko ya kgatelelo godimo ga histori ya dipolitiki tša Israele le Juta, gomme e be e le šedi ye kgolo ya boprofeta bja Jona le Nahume. Gape go be go na le Rehoboth Ir, Calah, le Resen. Calah (Akk. Kalkhu) e tsebega gabotse go rena lehono e le Nimrud, ye e lego dimaele tše e ka bago tše masomepedi borwa bja Ninife. Ge e le Resen, e tsebega ka tlhalošo ya bjale ya 'magareng ga Ninife le Calah' (Mathews 2001:450).

Go tšwa tshedimošong ye e filwego ka mo godimo, go laetša gore mafelo ao a mane a be a bopile toropo ye kgolo ya diriparipa, ditoropo tša mokoloko tšeo go bego go šomišwa leina la toropo ye kgolo (ya go tsebega) ya Ninife, go fapana le Ninife ye Nnyane, yeo Nimirode a ka bago a ikopantše le mafelo a mangwe a mararo go bopa mošate wo mogolo, le go ba sebo se segolo sa mmušo wa gagwe mo nokeng ya Thikirisi (Mathews 2001:450; Keil \& Delitzsch 2002:105-106).

\section{Thathollo ya mmakgonthe ya Thogako go Hama/Kuše /Nimirode}

Le ge boradithuto ka kakaretšo ba sentše sebopego sa Nimirodebjalo ka moloko wa Hama yo a rogakilwego, gomme ba bea thogako ye godimo ga Bathobaso ntle le tšhetšo, re kgona go lemoga tše di latelago go tšwa taodišwaneng ya rena (Gen. 10:8-12). Go bohlokwa go lemoga gore, go fapana le tše boradithuto ba mmalwa ba emego ka tšona, temana ya Bibele ye re swaraganego le yona ga e bone Nimirode ka tsela ya go befa le gatee. Gabotse, e šupa go seswantsho se sebotse sa dikatlego tša gagwe.
Sa mathomo, Nimirode o hlalošwa ka go Gen 10:9 e se mmušanoši, bjalo ke ge basodi ba gagwe ba laeditše, eupša bjalo ka mohlabani yo maatla, mogale mo lefaseng. Go feta fao, lentšu le 'maatla' mo ga se la šomišwa ka tsela ya tsholo.

Sa bobedi, o hlalošwa ka go Genesi 10:9 e le motsomi yo maatla pele ga Morena. Go ya ka taodišwana ye Nimirode ga a hlološwe e le lerabele mo eupša e le motsomi yo a bego a na le mabokgoni a go tsoma a a bego a feta ba meloko ya Seme le Jafete. Go tlaleletša fao, o laeditše bogolo bja mabokgoni pele ga Morena gomme ga go sešupo temaneng ye gore Morena o be a le kgahlanong le botsebi bjoo bja Nimirode.

Sa boraro, Nimirode o hlalošwa e le moagi wa ditoropo tše mmalwa. Se se laetša gore le ge rakgolo'agwe a be a rogakilwe gore e be lekgoba, Nimirode o gana go ikobela go išwa fase bophelong bja gagwe, ka go ithuta bokgoni bja go aga bjoo ka morago bo mo dirago moatšhitheke wa go tuma.

Sa bone, go fapana le boradithuto ba Mpshafatšo le Tsošološo, Genesi 10 ga e ukame gore go na le tswalano ka madi magareng ga thogako ya Hama le dikatlego tša Nimirode.

Sa bohlano, ge Nimirode a bitšwa lerabele, ditiro tša gagwe tša borabele di swanetše go amanywa le kabelo ya go se be ya toka le thogako ya rakgolo'agwe, Hama. Ka mantšu a mangwe, Nimirode o lwantšhana le thogako ya Noage godimo ga gagwe ka go itsoša, pele ga Morena, e le mmuši wa bokgoni, motsomi le moagi yo maatla.

Sa boselela, thogako ya Hama e be e se go fularelwa ke meloko ya gagwe go balwa le Nimirode, eupša e be e le maitshwaro a boitefetšo bjalo ka tate wa go hloka maikarabelo kgahlanong le morwa wa gagwe yo monnyane.

Sa bošupa Nimirode o ganana le mabaka a a mo emetšego ka dintlha tša menwana ka lebaka la thogako ya gagwe ka go šoma ka maatla a magolo, go se lape, go gapeletša le maikemišetšo. Go ya ka taodišwana ye Nimirode o swanetše go bonwa bjalo ka motho yo a lwelego le 'kganyogelo ya madimabe' ya boitefeletšo ya rakgolokhukhu'agwe, Noage, yo a 'rogakilego' Hama le meloko ya gagwe go tlo ba bahlankedi ba Seme le Jafete. Tabataba, ke gore go bile le ka fao Nimirode a lwelego le maemo a mabe gore e se be lekgoba, eupša moagi wa mabonwa, mmuši le motsomi wa bokgoni le tsebo.

Jamieson o tloga a opile phala mmala, ge a re Nimirode:

...o tloga a itšhupile ka go ba bogale le katlego ya maatla mo go tsomeng diphoofolo tša naga. Ka baka la mediro ye ya go retega o ile a ikhumanela leina a lebogwa ke batho; gomme, ka go ipea molaodi wa batho wa go ya go ile, e ile a hloma mmušo wa mathomo wa lefase [Gen 10:10].

(Keil \& Delitzsch 2002:105-106)

Go bonala gore Modimo o ile a ema le Nimirode ka go mo kgontšha go fihlelela dikatlego tše di boletšwego ka mo godimo ka go ganetšana le thogako ye e lebantšwego go rakgolo'agwe ke Noage. 
Sa boseswai, ke gopola gore re hloka go sekaseka ditiro tša Noage tšeo di hlotšego thogako ya Hama le Nimirode. Temana ya Sehebere e ukama gore Noage o itlhobotše ka boomo ka tsela ya go leša dihlong. Ba bangwe ba emelela Noage ka go šišinya gore maemo a atmosfere a lefase ka morago ga meetsefula a ile a hlola go bela ga morara, le gore Noage o be a sa tsebe ka botlalo se a bego a se dira (Wiersbe 1993). Eupša Noage a ka se no leswa a sa tšee maikarabelo a ditiro tša gagwe. Ke tate yo ditiro tša gagwe di ka bego di ile tša tlaiša bana ba gagwe. Sebakeng sa go tšea maikarabelo a maitshwaro a go se hlamatsege ka ditiro tša gagwe, o rogaka Hama, morwa wa gagwe yo monnyane ge a sa amogele maitshwaro a gagwe a go se thabiše.

\section{Phetho}

Kanegelo ya thogako ya Hama yeo e šupago gape le Nimirode, ga se ya šomišwa fela go tima Bathobaso ditokelo tša bona tša motheo, eupša e šomišitšwe le go nyatša dikakanyo le dikgopolo tša bona. Bjalo ka barogakwa ba moloko wa Hama, sa bona e be e swanetše go ba bahlankedi ba beng ba bona ba Makgowa. Eupša ge re lebelela kanegelo ya Nimirode, re bona motho yo a ilego a ithagaraga dilo di le bothata ka lebaka la thogako gore a atlege. Le ge e be e le wa 'lešika la thogako', Nimirode o ile a lwa le maemo ao gore e be motsomi yo maatla, mmuši le moagi. Nimirode ga se a dumelela 'thogako' go mo ema pele go fihlelela katlego mo modirong wa gagwe. Go fapana le seo o ile a swanela go šoma ka maatla (motsomi, moagi, mmuši) go itšhupa gore ke se sengwe setšhabeng seo go bego go le bao ba šegofaditšwego go feta thogako ya gagwe.

Go swana le Nimirode, 'barogakwa' ba Bathobaso ba Afrika-Borwa ba ile ba swanela go ithagaraga kgahlanong le mathata go hwetša ditokelo tša thuto, tša boiphedišo le tša sepolitiki. Le ge melao ye mmalwa e ile ya hlangwa ke mmušo wa kgethollo wa Afrika-Borwa, go gatelela le go šitiša Bathobaso, ba ile ba swanela go šoma ka maatla go lwa le pušo yeo ya kgatelelo ye e bego e se na taba le ditokelo tša bona tša motheo le tokologo. Maafrika-Borwa mono gae le moše a ile a lwantšhana le kgatelelo ya ona (a thušwa ke ditšhaba tša mafase), ye e bego e theilwe godimo ga kgopolo ya thogako ya Hama go setšhaba sa Bathobaso ka mekgwa ya go fapana ya boipelaetšo. Borathutatumelo e be e le ba bangwe ba ba etilego pele ka go gana taba ya go swantšha thogako ya Hama le Bathobaso ka Afrika-Borwa.

Taodišwana ye e laeditše gore go fapana le bao ba lebelelago Nimirode ka leihlo la go se be botse, Nimirode o swanetše go fiwa le tshekatsheko ya mmakgonthe ye a e hlokago kudu. Go Bathobaso ba ba phetšego mengwaga ye mentši ka tlase ga mabaka a thogako, bohlankedi le kgatelelo, Nimirode o swanetše go ba seka sa tlhohleletšo go ya ka fao a šomilego ka gona kgahlanong le mabaka a thata a thogako, gore e be motho yo a atlegilego ka kudu bophelong.

\section{Methopo}

Augustine, 1955, 'City of God, XVI:3', in P. Schaff (ed.), A selected Library of Nicene and Post Nicene Fathers of the Christian Church, 8 vols., Eerdmans, Grand Rapids.

Carson, D.A., 1994, New Bible commentary, 21st century edition, Inter-Varsity Press, Leicester and Downers Grove.

Clifford, R.J., 1985, 'Nimrod', in P.J Achtemeier (ed.), Society of Biblical Litreture. Harper's Bible dictionary, 1st edn., Harper \& Row, San Francisco.

Easton, M., 1996, Easton's Bible dictionary, Logos Research, Systems Oak Harbor.

Gero, S., 1980, 'The Legend of the Fourth Son of Noah', Harvard Theological Review 73, 321-30. http://dx.doi.org/10.1017/S0017816000002200

Gibson, J.C.L., 2001, Genesis: The Daily study Bible Series, vol. 1, John Knox Press, Louisville.

Gillespie, G.T., 1954, 'A Christian View of Segregation', an address made before the Synod of Mississippi of the Presbyterian Church in the US, Association of Citizens' Councils of Mississippi, Greenwood, 04 November.

Ginzberg, L., 1968, The Legends of the Jews, vol. 1, Jewish Publication Society of America, Philadelphia.

Haynes, S., 2002, Noah's Curse: The Biblical Justification of America Slavery, University Press, Oxford.

Henry, M., 1996, Matthew Henry's commentary on the whole Bible: Complete and unabridged in one volume, Hendrickson, Peabody.

Hughes, R.B., Laney, J.C. \& Hughes, R.B., 2001, Tyndale concise Bible commentary, rev. edn. of New Bible companion, 1990, includes index, The Tyndale reference library, Tyndale House Publishers, Wheaton.

Jamieson, R., Fausset, A.R., Fausset, A.R., Brown, D. \& Brown, D., 1997, A commentary, critical and explanatory, on the Old and New Testaments, Logos Research Systems, Oak Harbor.

Keil, C.F. \& Delitzsch, F., 2002, Commentary on the Old Testament, vol. 1, Hendrickson, Peabody.

Kenneth, R.K., 1956, 'The Segregation Issue', Baptist Bulletin, October, 9-10.

Kugel, J.L., 1997, The Bible as it was, Harvard University Press, Cambridge.

Luther's works, 1955-1986, 55 vols., ed. J. Pelikan, Cancordia, St. Louis.

Mathews, K.A., 2001, Genesis 1-11:26 (electronic ed.), Logos Library System, The New American Commentary, vol. 1A, Boardman and Holman Publishers, Nashville.

Oded, B., 1986, 'The Table of Nations (Genesis 10)- A Sociocultural Approach', ZAW 98,31 .

Pennington, J.W.C., 1841, A Text Book of the Origin and History of the Colored People, L. Skinner, Hartford.

Richards, L., 1991, The Bible reader's companion, includes index, Victor Books, Wheaton.

Sandell, J.W., 1907, The United States in Scripture. The Union Against the States. God in Government, Tucker Printing House, Mississippi.

Walvoord, J.F., Zuck, R.B. \& Dallas Theological Seminary, 1985, The Bible knowledge commentary: An expositin of the scriptures, vol. 1, Victor Books, Wheaton.

Wiersbe, W.W., 1993, Wiersbe's expository outlines on the Old Testament, Victor Books, Wheaton.

Wiseman, D.J.N, 1996, in D.R.W. \& I.H. Marshall, New Bible Dictionary, includes Index, (electronic edn. of 3rd edn.), Inter Varsity Press, Downers Grove.

Williams, D.K., 2004, An End to This Strife: The Politics of Gender in African American Churches Fortress Press, Minneapolis.

Willmington, H.L., 1997, Willmington's Bible Handbook, Tyndale House Publishers, Wheaton.

Wittenberg, G., 1991, “'Let Canaan be his slave” (Genesis 9:26) Is Ham also cursed?', Journal of Theology for Southern Africa, 46-56. 